\title{
Composición y diversidad de la regeneración natural en comunidades de Pinus- Quercus sometidas a una alta recurrencia de incendios en el noreste de México
}

\section{Composition and diversity of natural regeneration in Pinus-Quercus communities under high fire recurrence in northeastern Mexico}

\author{
Eduardo Alanís-Rodríguez ${ }^{1 凶}$, Javier Jiménez-Pérez ${ }^{1}$, Alejandro Valdecantos-Dema ${ }^{2}$, Marco A. González- \\ Tagle ${ }^{1}$, Óscar A. Aguirre-Calderón ${ }^{1}$ y Eduardo J. Treviño-Garza ${ }^{1}$ \\ ${ }^{I}$ Facultad de Ciencias Forestales, Universidad Autónoma de Nuevo León. Carretera Linares-Cd. Victoria Km. 145. Apartado postal 41, 67700 Linares, \\ Nuevo León, México. \\ ${ }^{2}$ Fundación de la Comunidad Valenciana. Centro de Estudios Ambientales del Mediterráneo (CEAM), Unidad Mixta CEAM-UA. Parque Tecnológico, \\ c/ Charles R. Darwin 14, Valencia, España. \\ \eduardo.alnisrd@uanl.edu.mx
}

\begin{abstract}
Resumen. Se estudió la regeneración natural a medio plazo de especies leñosas en un bosque mixto de Pinus-Quercus sometido a alta recurrencia de incendios (1972, 1984 y 1998) de mediana-alta intensidad en el noreste de México. El objetivo fue determinar la capacidad de recuperación de la vegetación frente a incendios recurrentes, y si ésta se puede ver modificada por una variable fisiográfica como es la exposición de la ladera (NE vs. NO). Los resultados mostraron que el 93\% de las especies arbóreas son rebrotadoras obligadas que ya estaban antes del fuego. Esta capacidad de rebrote permite la reconstitución de la parte aérea de individuos ya existentes. El género clave por su abundancia es Quercus, siendo Q. rysophylla la especie más abundante después del fuego. Si bien la especie Pinus pseudostrobus está adaptada al fuego mediante una corteza gruesa y la longevidad de su banco de semillas, los incendios de medianaalta intensidad de manera frecuente implican la pérdida local de la especie. Las exposiciones evaluadas no presentan diferencias significativas en su densidad $(\mathrm{N} / \mathrm{ha})$ y dominancia $\left(\mathrm{m}^{2} / \mathrm{ha}\right)$ pero sí en su diversidad y composición, siendo la exposición NO la más diversa. Estos resultados proporcionan una herramienta a considerar en la elaboración de planes de gestión de áreas protegidas del NE de México.
\end{abstract}

Palabras clave: diversidad, exposición de ladera, Pinus pseudostrobus, Quercus spp., riqueza específica.

\begin{abstract}
We evaluated the natural regeneration of woody species at the mid-term in a Pinus-Quercus mixed forest affected by frequent medium-to-high intensity wildfires (1972, 1984 and 1998) in northeastern Mexico. The objective was to determine the recovery of vegetation from recurrent fires, and whether it depends on slope exposure (NE vs. NW). The results indicated that $93 \%$ of the species are obligated resprouters already present in the site before the fires. This capability enables the reconstitution of aerial part of existing individuals. According to abundance records, the key genus was Quercus, and Q. rysophylla was the most abundant species after the fires. While the specie Pinus pseudostrobus is adapted to fire due to a thick bark and the long life span of its seed bank, frequent medium-to-high intensity fires implied the local extinction of the species. We did not find significant differences in density (N/ha) and dominance $\left(\mathrm{m}^{2} / \mathrm{ha}\right)$ in relation to the exposures assessed, but in terms of diversity and composition there were significant differences, being the NW the most diverse exposure. These results provide useful information to be considered for the development and design of management plans of protected areas in NE Mexico.
\end{abstract}

Key words: diversity, exposure, Pinus pseudostrobus, Quercus spp., species richness.

\section{Introducción}

El fuego es un factor natural en muchos ecosistemas y tiene una influencia importante en la productividad biológica y composición de la vegetación (Iniguez et al., 2008). Después de los incendios se crean espacios abiertos por la eliminación de la vegetación, los cuales favorecen la ger-

Recibido: 31 enero 2012; aceptado: 24 mayo 2012 minación y rebrote de individuos de especies pre-existentes o presentes en el banco de semillas del suelo (Calvo et al., 2008; Weiguo et al., 2008; Vidal y Reif, 2011). En muchos ecosistemas boscosos los incendios forestales son vitales para la renovación y rejuvenecimiento de sus elementos vegetales (Rodríguez, 2008). Además de estos efectos directos del fuego sobre la vegetación de un ecosistema, existen otras consecuencias de los incendios forestales sobre diferentes componentes y procesos ecológicos de los 
mismos. Por una parte, el suelo queda desprotegido por la pérdida de la vegetación y junto con un aumento de la hidrofobicidad y del sellado del suelo, pueden producirse procesos erosivos que lleven a la pérdida y exportación de suelo y sedimentos (Woods et al., 2007), y por la otra, pueden producirse cambios transitorios en la disponibilidad de nutrientes y en el pH del suelo debidos a la volatilización y a la deposición de cenizas (Certini, 2005). Es factible que estos efectos determinen los procesos sucesionales post-incendio y en consecuencia, condicionen la estructura vegetal de las comunidades resultantes.

Las tormentas eléctricas y erupciones volcánicas son las causas naturales más frecuentes de los incendios forestales en el mundo (Villarreal y Yool, 2008). Sin embargo, la recurrencia de los incendios ha sido crecientemente modificada por la acción humana, a través de la intervención cada vez más agresiva sobre los recursos naturales (González et al., 2005; Jiménez y Alanís, 2011). En áreas donde los incendios se producían por mecanismos naturales, el ser humano ha intervenido los ecosistemas y ha excluido el fuego y alterado el proceso, lo que ha provocado cambios considerables en la estructura y composición de especies (Fulé y Covington, 1999; Fulé et al., 2005) así como en la acumulación de combustible y en el riesgo de incendios de alta intensidad cuando éstos finalmente se producen (Scott y Reinhardt, 2001).

En los ecosistemas boscosos del noreste de México los incendios forestales se distribuyen ampliamente (Yocom et al., 2010; Jiménez y Alanís, 2011). No obstante, los estudios ecológicos sobre cómo el fuego afecta las especies vegetales son pocos (Rodríguez-Trejo y Fulé, 2003; Martínez y Rodríguez, 2008; Rodríguez-Trejo y Myers, 2010). Debido a la escasa información sobre la ecología de las especies presentes en los ecosistemas mencionados, resulta importante estudiar cómo se produce la regeneración de la vegetación afectada por incendios recurrentes y cómo son los ecosistemas resultantes desde el punto de vista de su composición (Alanís et al., 2011). Las especies vegetales pueden clasificarse por su respuesta regenerativa post-incendio en especies rebrotadoras y especies germinadoras, según tengan o no la capacidad de rebrotar y germinar después de ser quemadas por completo (Pausas, 2004). Los ecosistemas de Pinus-Quercus del NE de México se caracterizan por estar expuestos a condiciones climáticas extremas, donde la disponibilidad de agua es un factor limitante (Himmelsbach et al., 2010). Por esta razón, la orientación de las laderas dominantes tiene una importancia notable en las condiciones microclimáticas, modificando la evapotranspiración potencial y la humedad edáfica (van de Water et al., 2002). En este sentido, Konstantinidis et al. (2006) observaron cómo el vigor y el desarrollo de los rebrotes tras incendio forestal en el
Mediterráneo era significativamente mayor en laderas con orientación norte y este que en orientaciones sur y oeste. González et al. (2008) evaluaron la regeneración de la vegetación post-incendio en un ecosistema del noreste de México, donde determinaron que la exposición norte presentaba mayor densidad y área basal.

Por otro lado, tiene especial interés conocer el efecto de la variación ambiental en dicha regeneración, especialmente el efecto de la variación microclimática, lo que permitiría determinar áreas con mayor o menor potencial de regeneración y, por tanto, con mayor o menor requerimientos de manejo. Por lo anterior, el conocimiento de la autoecología y de las estrategias regenerativas de las especies vegetales presentes en un ecosistema es fundamental para entender los procesos de recuperación del ecosistema ante incendios recurrentes y prever sus posibles consecuencias. El empleo de este tipo de información se ha incrementado entre los científicos, técnicos y gestores de sistemas naturales, ya que es el punto de partida para la correcta toma de decisiones dentro de los programas de rehabilitación y restauración ecológica (Alanís et al., 2010).

Con este planteamiento, los objetivos generales de la presente investigación fueron: 1) catalogar las especies arbóreas ( $\geq 1 \mathrm{~cm}$ de diámetro) por su respuesta regenerativa en un bosque mixto de Pinus-Quercus sometido a alta recurrencia de incendios forestales; 2) estimar los parámetros ecológicos de abundancia $\left(\mathrm{AR}_{\mathrm{i}}\right)$, dominancia $\left(\mathrm{DR}_{\mathrm{i}}\right)$, frecuencia $\left(\mathrm{FR}_{\mathrm{i}}\right)$ e índice de valor de importancia (IVI), 3) caracterizar el estrato arbóreo mediante la determinación de la densidad de individuos y el área de copa; 4) cuantificar la diversidad $\alpha$ y $\beta$ y 5) analizar si estas variables pueden ser modificadas por una variable fisiográfica, como es la exposición de la ladera (NE vs. NO). La hipótesis de partida es que la vegetación arbórea presentará mayor abundancia y dominancia en la exposición noreste. La información generada profundizará en el conocimiento ecológico de las especies frente al fuego y proporcionará importantes elementos a considerar en la toma de decisiones sobre la gestión de estos ambientes.

\section{Materiales y métodos}

El área de estudio se localiza en el Parque Ecológico Chipinque (municipios de San Pedro Garza García y Monterrey, Nuevo León, México (Fig. 1), el cual forma parte del Área Natural Protegida Parque Nacional Cumbres de Monterrey. La altitud de la zona de estudio varía de los 1 100 a los $1150 \mathrm{~m}$ snm, situándose entre las coordenadas geográficas $25^{\circ} 33^{\prime}-25^{\circ} 35^{\prime} \mathrm{N}, 100^{\circ} 18^{\prime}-100^{\circ} 24^{\prime} \mathrm{O}$. La vegetación de un ecosistema maduro de la zona corresponde a un bosque mixto conformado por especies de los géneros 
Pinus y Quercus, entre las que destacan Pinus pseudostrobus Lindl. (especie clave por su alta densidad y cobertura en ecosistemas maduros de referencia), Quercus rysophylla Weath, $Q$. laeta Liemb, $Q$. polymorpha Schltdl. y Cham., $Q$. laceyi Small y Q. canbyi Trel. (Jiménez et al., 2001).

La zona de estudio ha sufrido una alta recurrencia de incendios de intensidad media-alta en los últimos 40 años, con fuegos en los años 1972, 1984 y 1998 (González et al., 2007). En julio de 2008, 10 años después del último incendio forestal, se establecieron 2 áreas de estudio en las que se caracterizó la comunidad vegetal regenerada tras el fuego. Ambas áreas presentaban similitud de características geográficas (Cuadro 1), partían de un ecosistema similar antes del primer episodio de fuego y se presentan sobre suelo litosol, pero difieren en exposición ( $\mathrm{NE} v s$. NO), aspecto que fue objeto de evaluación.

En cada zona se establecieron 4 parcelas de muestreo cuadradas de $100 \mathrm{~m}^{2}$, con una equidistancia de $20 \mathrm{~m}$ en las que se evaluaron las especies leñosas. En las parcelas de muestreo se realizó un inventario de las especies presentes y se midió el diámetro basal $\left(d_{0.10}\right)$ y el área de copa de los individuos de las especies del estrato arbóreo con un diámetro $\geq 1 \mathrm{~cm}$. La medición del diámetro se efectuó a $0.10 \mathrm{~m}$ sobre la base del suelo debido a las múltiples bifurcaciones que presentaron los individuos a mayor altura.

A partir de los datos de los sitios de muestreo, se derivó información fitosociológica y dasométrica. Para cada especie se determinó su abundancia, de acuerdo con el número de árboles, su dominancia, en función del área basal, y su frecuencia con base en su existencia en los sitios de muestreo. Los resultados se utilizaron para obtener un valor ponderado de taxón denominado Índice de Valor de Importancia (IVI), que adquiere valores porcentuales en una escala del 0 al 100 (Müller y Ellemberg, 1974). Para estimar la riqueza de especies se utilizó el índice de Margalef $\left(\mathrm{D}_{\mathrm{Mg}}\right)$ (Clifford y Stephenson, 1975) y para la diversidad de especies el índice de Shannon y Weiner $\left(\mathrm{H}^{\prime}\right)$ (Shannon, 1948). La diversidad $\beta$ se evaluó con base al coeficiente de similitud de Sorensen para datos cuantitativos (Magurran, 2004).

Las especies registradas en la investigación se catalogaron de acuerdo con la clasificación propuesta por Pausas

Cuadro 1. Características generales de las 3 áreas evaluadas

\begin{tabular}{lcc}
\hline & $N O$ & $N E$ \\
\hline Latitud (N) & $25^{\circ} 36^{\prime} 03^{\prime \prime}$ & $25^{\circ} 36^{\prime} 02^{\prime \prime}$ \\
Longitud (O) & $100^{\circ} 20^{\prime} 10^{\prime \prime}$ & $100^{\circ} 20^{\prime} 27^{\prime \prime}$ \\
Elevación (m snm) & 1235 & 1210 \\
Pendiente $\left({ }^{\circ}\right)$ & 33 & 36 \\
Proximidad de propágulos $(\mathrm{m})$ & 240 & 170 \\
\hline
\end{tabular}

(2004) y Pausas et al. (2004), dividiéndolas en 4 estrategias principales para las plantas que aparecen después del fuego, según su capacidad de rebrotar y la resistencia de sus propágulos (Cuadro 2; Pausas, 2004).

Aplicamos análisis de componentes principales (PCA) para explorar la estructura de los datos de las distintas parcelas experimentales en función de la abundancia y dominancia de las especies leñosas (14 variables en cada caso). Para determinar si la orientación de la ladera modificaba significativamente la riqueza, diversidad, abundancia y dominancia (área basal) de las especies, se realizó un análisis de varianza ANOVA de un factor (orientación) a 2 niveles ( $\mathrm{NO}$ y NE). Los datos fueron transformados logarítmicamente para homogeneizar las varianzas. Todos los análisis se realizaron mediante el paquete estadístico SPSS versión 15.0 (SPSS Inc., Chicago, Il, USA).

\section{Resultados}

Se registraron 9 familias, 10 géneros y 14 especies leñosas (Cuadro 3). La familia con mayor presencia fue Fagaceae con 4 especies. El género con mayor presencia fue Quercus con las especies $Q$. canbyi, Q. laeta, $Q$. laceyi y $Q$. rysophylla. De las 14 especies registradas y de

\section{LOCALIZACIÓN PARQUE ECOLÓGICO CHIPINQUE, A.C.}

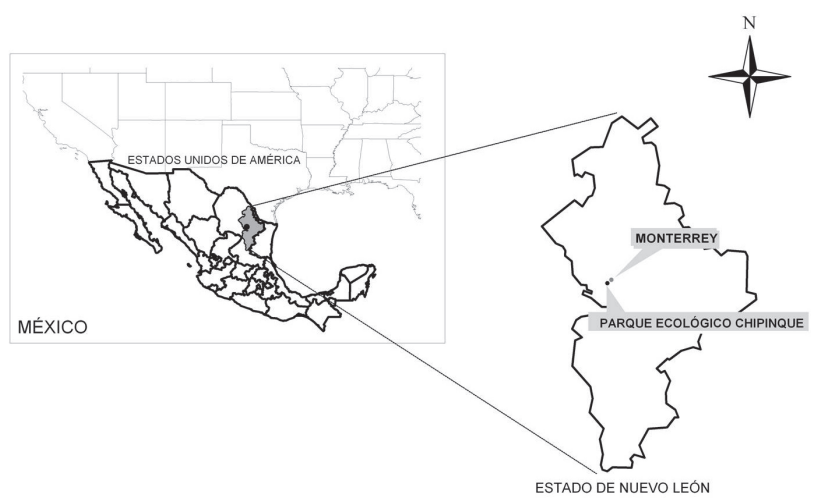

Figura 1. Localización de la zona de estudio.

Cuadro 2. Clasificación de las especies vegetales en función del tipo de regeneración post-incendio (Pausas, 2004)

\begin{tabular}{cccc}
\hline & \multicolumn{2}{c}{ Banco de semillas } \\
& SI & $\begin{array}{c}\text { Rebrotadoras } \\
\text { facultativas }\end{array}$ & $\begin{array}{c}\text { Rebrotadoras } \\
\text { obligadas }\end{array}$ \\
$\begin{array}{c}\text { Capacidad de } \\
\text { rebrotar }\end{array}$ & NO & $\begin{array}{c}\text { Germinadoras } \\
\text { obligadas }\end{array}$ & $\begin{array}{c}\text { Germinadoras } \\
\text { colonizadoras }\end{array}$ \\
\hline
\end{tabular}


Cuadro 3. Estrategia regenerativa (Est. reg.), densidad y área basal (media y error típico de las 4 parcelas por orientación) e Índice de Valor de Importancia (IVI) de las especies registradas en función de la orientación de la ladera

\begin{tabular}{|c|c|c|c|c|c|c|c|}
\hline \multirow{2}{*}{ Especies } & \multirow{2}{*}{$\begin{array}{l}\text { Est. } \\
\text { reg. }\end{array}$} & \multicolumn{2}{|c|}{ Densidad (N/ha) } & \multicolumn{2}{|c|}{ Área basal $\left(m^{2} / h a\right)$} & \multicolumn{2}{|c|}{ IVI } \\
\hline & & NO & $N E$ & NO & $N E$ & NO & $N E$ \\
\hline Arbutus xalapensis Kunth. & RO & $25 \pm 25$ & 0 & $0.03 \pm 0.30$ & $0.00 \pm 0.00$ & 1.55 & 0 \\
\hline Ceanothus coeruleus Lag. & RO & $325 \pm 144 a$ & $\mathbf{0 b}$ & $0.30 \pm 0.20$ & $0.00 \pm 0.00$ & 6.72 & 0 \\
\hline Celtis laevigata $\mathrm{L}$. & RO & 0 & $75 \pm 48$ & $0.00 \pm 0.00$ & $0.08 \pm 0.07$ & 0 & 3.35 \\
\hline Cercis canadensis $\mathrm{L}$. & RO & 0 & $75 \pm 48$ & $0.00 \pm 0.00$ & $0.25 \pm 0.14$ & 0 & 3.58 \\
\hline Chiococca pachyphylla Wernh. & RO & $50 \pm 29$ & $25 \pm 25$ & $0.01 \pm 0.00$ & $0.18 \pm 0.04$ & 3.04 & 1.79 \\
\hline Juglans mollis Hemsl. & RO & 0 & $325 \pm 263$ & $0.00 \pm 0.00$ & $0.80 \pm 0.60$ & 0 & 5.89 \\
\hline Pinus pseudostrobus Lindl. & GO & 0 & $50 \pm 29$ & $0.00 \pm 0.00$ & $0.01 \pm 0.01$ & 0 & 1.72 \\
\hline Prunus mexicana S. Wats. & RO & 0 & $100 \pm 71$ & $0.00 \pm 0.00$ & $0.60 \pm 0.37$ & 0 & 4.23 \\
\hline Prunus serotina Ehrenb. & RO & $25 \pm 25 a$ & $225 \pm 85 b$ & $0.26 \pm 0.26$ & $0.54 \pm 0.22$ & 1.84 & 6.31 \\
\hline Rubus trivialis Michx. & RO & 0 & $25 \pm 25$ & $0.00 \pm 0.00$ & $0.02 \pm 0.01$ & 0 & 1.57 \\
\hline Quercus canbyi Trel. & RO & $975 \pm 75 A$ & $150 \pm 119 B$ & $7.01 \pm 3.21 \mathrm{~A}$ & $0.50 \pm 0.48 B$ & 22.47 & 4.39 \\
\hline Quercus laeta Liemb. & RO & $350 \pm 119 A^{*}$ & $\mathbf{0 B} *$ & $0.81 \pm 0.44 a$ & $0.00 \pm 0.00 b$ & 8.88 & 0 \\
\hline Quercus laceyi Small. & RO & $1000 \pm 91$ & $1000 \pm 308$ & $5.82 \pm 2.87$ & $2.54 \pm 0.93$ & 21.17 & 15.24 \\
\hline Quercus rysophylla Weath. & RO & $1850 \pm 638$ & $3325 \pm 798$ & $12.50 \pm 4.24$ & $18.74 \pm 0.74$ & 34.32 & 51.93 \\
\hline Total & & $4600 \pm 981$ & $5375 \pm 2056$ & $26.74 \pm 5.26$ & $24.25 \pm 2.20$ & 100 & 100 \\
\hline
\end{tabular}

NO, noroeste; NE noreste. RO, rebrotadora obligada; GO, germinadoras obligadas. Negritas y con letras diferentes para cada una de las orientaciones, indican diferencias significativas (mayúsculas $p<0.05$; minúsculas $0.05<p<0.1$ ).

* Varianzas no homogéneas.

acuerdo a la clasificación de Pausas (2004), 13 presentan como estrategia regenerativa rebrotar y 1 exclusivamente es germinadora obligada.

Especies rebrotadoras. De acuerdo con el Índice de Valor de Importancia (IVI) el género con mayor representatividad fue Quercus, presentando en el área NO un $89 \%$ de representatividad y en el área NE un 72\%. La exposición NO presentó 8 especies rebrotadoras obligadas, siendo $Q$. rysophylla, $Q$. canbyi y $Q$. laceyi las de mayor peso ecológico. En la exposición NE se encontraron 11 especies rebrotadoras obligadas, siendo $Q$. rysophylla y $Q$. laceyi las de mayor peso ecológico. Cabe destacar que las áreas evaluadas están constituidas por pocas especies que presentan altos valores de densidad. Así, en la exposición NE, 2 especies (Q. rysophylla y Q. laceyi) representan el $80 \%$ de la abundancia relativa. Encontramos especies que sólo están presentes en 1 de las laderas evaluadas. Así, la ladera de orientación NO alberga individuos de Arbutus xalapensis, Ceanothus coeruleus y $Q$. laeta que están ausentes de la orientación NE. Por el contrario, en la ladera NE encontramos Celtis laevigata, Cercis canadensis, Juglans mollis, Prunus mexicana y Rubus trivialis, cuya presencia no se registró en la ladera NO.

Especies germinadoras. Pinus pseudostrobus fue la única especie que una vez quemada la parte aérea por completo no rebrotó. Dicha especie sólo se encontró en la exposición
NE, presentando una baja densidad (50 individuos/ha) y área basal $\left(0.01 \mathrm{~m}^{2} / \mathrm{ha}\right)$.

Abundancia y cobertura. En el Cuadro 3 se muestran los valores promedios de la abundancia y cobertura, sin que se observaran diferencias significativas entre orientaciones para ninguna de las variables $(\mathrm{F}=0.433$, g.l. $=7, p=$ 0.535 y $\mathrm{F}=0.908$, g.l. $=7, p=0.378$ para la densidad $\mathrm{y}$ área basal, respectivamente). Por lo tanto, se rechaza la hipótesis de partida. No obstante, se obtuvo un aumento del $15 \%$ en el número de individuos en la ladera NE respecto a la NO, si bien el área basal disminuyó en un $10 \%$ aproximadamente, sugiriendo menos individuos pero de mayor tamaño en la orientación NO. Para generar información más precisa, se realizó un análisis de las especies comunes en las 2 exposiciones, Chiococca pachyphylla, Prunus serotina, Quercus rysophylla y $Q$. laceyi no mostraron diferencia significativa en la densidad $(p>0.05)$, mientras que $Q$. canbyi presentó prácticamente 6 veces mayor densidad en la exposición $\mathrm{NO}$ que en la NE, siendo significativas estas diferencias $(\mathrm{F}=34.380$, g.1. $=7$, $p=0.001$ ) (Cuadro 2). Los datos de cobertura mostraron efectos similares a los de abundancia, si bien las diferencias entre orientaciones dejaron de ser significativas para algunas especies. Únicamente $Q$. canbyi presentó una abundancia significativamente mayor en $\mathrm{NO}$ que en $\mathrm{NE}$ $(\mathrm{F}=9.602$, g.l. $=7, p=0.021)$. 
Según el análisis de componentes principales (PCA) para los datos de densidad, las parcelas se separan claramente en función de su orientación (Fig. 2). El primer componente explica un $33.4 \%$ de la varianza (autovalor= 4.672) y el segundo un $22.1 \%$ adicional (autovalor= 3.097). Las parcelas en orientación NE y NO se distribuyeron en valores positivos y negativos del eje 1 , respectivamente. Las especies que más peso tienen en el primer eje son Prunus serotina, P. mexicana, Celtis laevigata, Cercis canadensis, Pinus pseudostrobus (éstas con valores positivos), Quercus canbyi, Q. laeta y Ceanothus coeruleus (valores negativos). Por su parte, abundancias elevadas de Chiococca pachyphylla se asocian con valores altos del segundo componente y Juglans mollis y Rubus trivialis con valores negativos. En cualquier caso, es el primer componente y, por tanto, las especies extraídas en él las que marcan la separación clara entre orientaciones.

Para los datos de dominancia, el PCA ofreció la misma clara separación entre orientaciones, si bien, mostró ciertos cambios respecto a la ordenación observada por abundancias en relación con el peso de las especies en cada componente. El primer componente, con un porcentaje de varianza explicada del $30.5 \%$, está asociado positivamente a la dominancia de Prunus mexicana, Cercis canadensis y Celtis laevigata, y negativamente con Quercus laceyi. Quercus canbyi, Prunus serotina y Ceanothus coeruleus se asocian con valores positivos del segundo componente ( $21.6 \%$ de varianza explicada), mientras que Juglans mollis y Rubus trivialis tienen un peso negativo en este eje. Al igual que se ha observado en el PCA de abundancias, es el primer componente el que separa las parcelas según su orientación (Fig. 3).

Riqueza y diversidad. De las 14 especies registradas en la investigación, 9 se presentaron en la exposición NO y 11 en la NE. Los resultados del índice de Margalef $\left(D_{M g}\right)$ coinciden con lo anterior, ya que para la orientación NO se registró un valor de $1.17 \pm 0.09$ y para la $\mathrm{NE}$ de $1.39 \pm 0.70$, sin que estas diferencias fueran estadísticamente significativas $(\mathrm{F}=0.386, \mathrm{~g} .1 .=7, p=0.557)$. De acuerdo con el índice de diversidad de Shannon $\left(H^{\prime}\right)$ el área NO presentó un valor de $1.35 \pm 0.04$ y la NE de $1.18 \pm 0.29$ (Fig. 4). El análisis de varianza determinó la ausencia de diferencias significativas en la diversidad del componente arbóreo entre ambas orientaciones $(\mathrm{F}=$ 1.426 , g.l. $=7, p=0.278)$. De acuerdo con el coeficiente de similitud de Sorensen para datos cuantitativos, las orientaciones evaluadas mostraron una similitud baja-media (44\%) presentando sólo 5 especies en común de las 14 registradas. Las especies que se establecieron en ambas exposiciones fueron Quercus rysophylla, $Q$. laceyi, $Q$. canbyi, Prunus serotina y Chiococca pachyphylla.

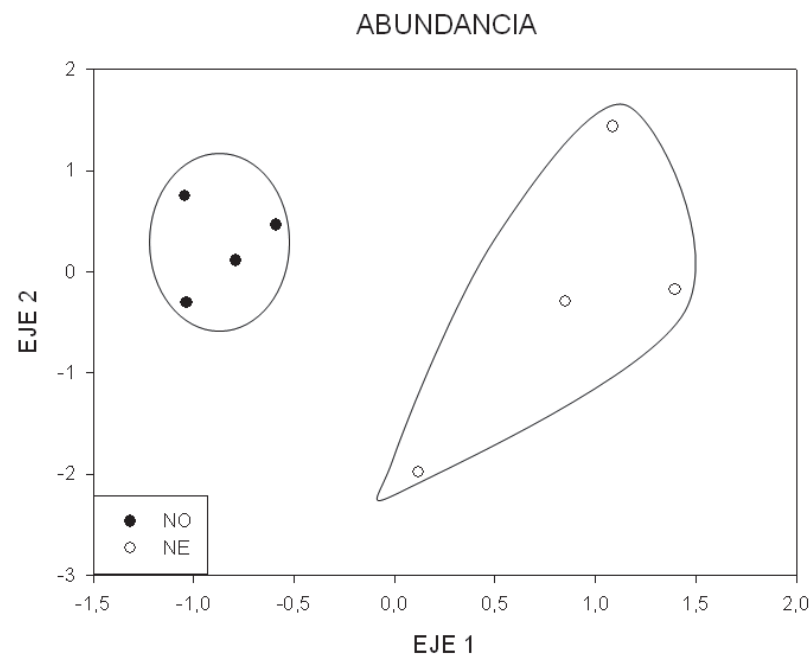

Figura 2. Distribución de las parcelas de estudio según su orientación (NO y NE) en los 2 primeros componentes generados por los datos de abundancia de las especies leñosas.

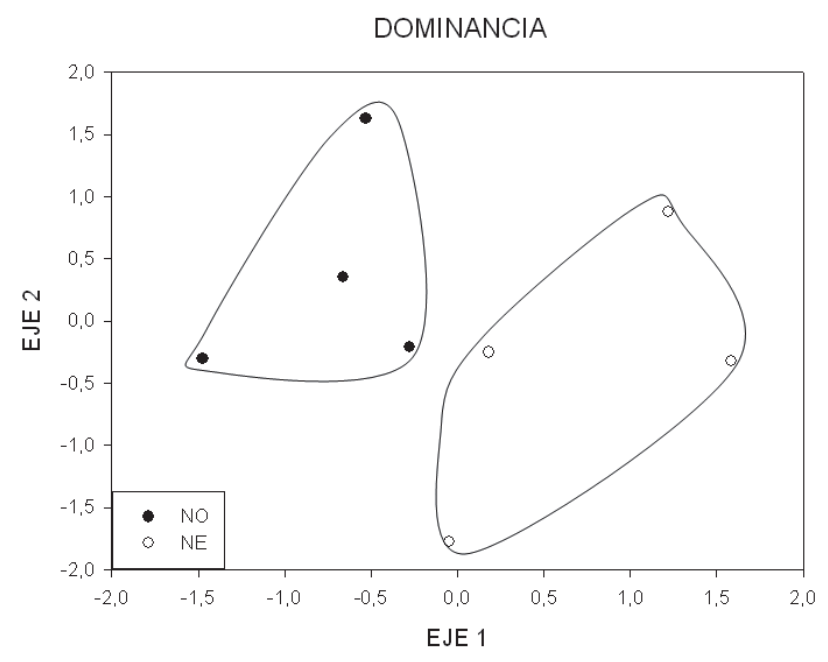

Figura 3. Distribución de las parcelas de estudio según su orientación (NO y NE) en los 2 primeros componentes generados por los datos de dominancia de las especies leñosas.

\section{Discusión}

De acuerdo con la clasificación propuesta por Pausas (2004) y Pausas et al. (2004), el 93\% de las especies leñosas registradas en la zona de estudio, 10 años después del último incendio, son rebrotadoras facultativas. La capacidad de respuesta de estas especies ante el fuego es responsable de la alta tasa de regeneración de la comunidad vegetal incendiada. Las especies que tienen como estrategia evolutiva rebrotar persisten al fuego, de manera que los individuos de estas especies presentes antes del 


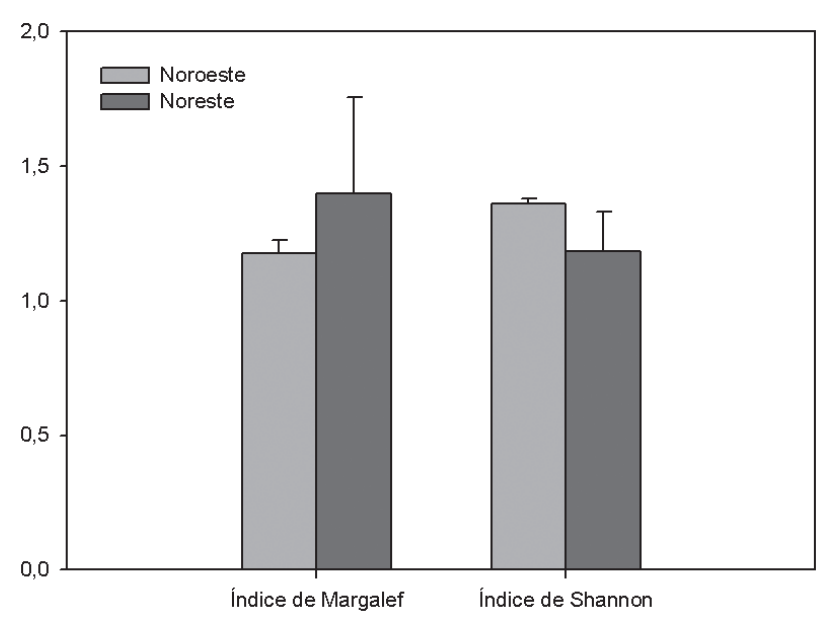

Figura 4. Valores de los índices de diversidad $\alpha$ de Margalef y Shannon (media y error típico) 10 años después del último incendio forestal en un bosque mixto de Pinus-Quercus en función de la orientación de la ladera.

incendio vuelven a implantarse en la zona incendiada (Pausas, 2004). En un ecosistema de Pinus-Quercus del noreste de México afectado por un incendio en 1998, García (2000) desarrolló un estudio en el cual cuantificó que $Q$. rysophylla y $Q$. canbyi presentaron 9.2 y 8.2 rebrotes por individuo, respectivamente, un año después de haber sufrido el impacto del incendio. Estos rebrotes representan una ventaja frente a las especies no rebrotadoras respecto al acaparamiento y uso de los recursos durante las primeras etapas post-incendio. En las áreas de estudio, objeto de la presente investigación, se registraron $2.15 \pm 1.79$ y $2.04 \pm$ 1.38 rebrotes por individuo de $Q$. rysophylla y $Q$. canbyi, respectivamente, 10 años después del incendio (datos no mostrados).

La única especie no rebrotadora que se registró fue Pinus pseudostrobus. Esta especie presenta como estrategia evolutiva la presencia de una corteza gruesa y resistente que protege al individuo en caso de incendios de baja intensidad (Rodríguez-Trejo y Fulé, 2003). Sin embargo, ante incendios de mayor intensidad los individuos se queman por completo y no persisten. No obstante, se ha registrado que esta especie genera un importante banco de semillas en el suelo (Marroquín et al., 2007). Estas semillas, debido a la alternancia de las temperaturas que se alcanzan en la superficie del suelo de un ecosistema incendiado (Baeza y Roy, 2008), germinan después de un incendio permitiendo la emergencia de nuevos individuos que forman una nueva población. La zona de estudio, al haber sido afectada reiteradamente por incendios de mediana y alta intensidad en las últimas décadas (González et al., 2007), probablemente presenta un banco de semillas poco abundante y de viabilidad reducida, generando una escasa germinación de esta especie. Sin embargo, Marroquín et al. (2007), en un estudio de un bosque de Pinus-Quercus en el noreste de México con el régimen de fuego natural, observaron el establecimiento de 582 plántulas de Pinus pseudostrous por hectárea, 3 años después de un incendio.

La persistencia de las poblaciones de especies no rebrotadoras en condiciones de incendios recurrentes es determinada por la longevidad del banco de semillas del suelo (Pausas et al., 2004), así como por sus ciclos vitales; dos incendios de mediana o alta intensidad distanciados por un periodo de tiempo menor al necesario para que la mayoría de los individuos lleguen a la edad adulta, implica la pérdida local de la especie (Pausas, 2004). En las últimas décadas, se han modificado la frecuencia e intensidad de incendios forestales; de manera natural se presentan cada 7.2 años y son superficiales (Jiménez y Alanís, 2011). Desde el año 1920 hubo políticas de supresión del fuego, lo que trajo como consecuencia la acumulación de combustible. Actualmente se presentan incendios de mediana y alta severidad con intervalos de 9.1 años (González et al., 2008). De manera que, a pesar de que las especies del género Pinus regeneran bien después de un incendio (Richardson, 2000), la alteración del intervalo y severidad ha eliminado temporalmente las poblaciones de Pinus pseudostrobus en diversas localidades de la sierra Madre Oriental (Alanís et al., 2008). En estos casos, el establecimiento de las especies germinadoras obligadas está condicionado por el tamaño del incendio, pues la aparición de nuevos individuos dependerá de la llegada de propágulos viables desde individuos semilleros situados en el perímetro del incendio. En el caso de esta investigación los individuos semilleros se encontraban a 0.8 kilómetros.

La densidad del componente arbóreo de las zonas de estudio osciló en torno a los 5000 individuos/ha con valores de área basal de $25 \mathrm{~m}^{2} /$ ha. Estos valores son mayores a los registrados por González et al. (2008), quienes observaron densidades de 3400 individuos/ha y área basal de $3.40 \mathrm{~m}^{2} /$ ha 4 años tras el fuego, poniendo de manifiesto que se siguen produciendo reclutamiento y establecimiento de rebrotes en el periodo comprendido entre los 4 y 10 años. No obstante, estos autores sólo tomaron en consideración individuos con diámetros superiores a $5 \mathrm{~cm}$., mientras que en el presente trabajo se consideraron todos los individuos de diámetro superior a $1 \mathrm{~cm}$.

Cabe destacar que la comunidad madura de PinusQuercus en el noreste de México presenta una densidad media de 340 individuos/ha (Jiménez et al., 2001). La elevada densidad de pies registrada en esta investigación puede relacionarse en parte con la capacidad que tienen las especies de Quercus presentes en el área de estudio, que además de rebrotar de la base del tronco, también rebrotan desde la raíz (obs. per.). Esta capacidad de respuesta ante 
el fuego es la responsable de la alta densidad registrada, siendo buena parte de los individuos presentes rebrotes de árboles maduros pre-existentes.

Hemos observado una clara separación de las parcelas en respuesta a su orientación dominante. En las parcelas orientadas a NE se encuentra una mayor densidad de las 2 especies de Prunus, Celtis laevigata, Cercis canadensis y presencia de la especie clave, y única germinadora, Pinus pseudostrobus, mientras que en las orientaciones NO abundan las quercíneas (Q. canbyi y Q. laeta). Estos resultados difieren de los de Otto et al. (2010), quienes no encontraron diferencia significativa en la densidad y cobertura de la regeneración arbórea en exposiciones de ladera norte y sur en bosques templados.

La riqueza específica total $(S=14)$ y por exposición $\left(S_{N O}=9, S_{N E}=11\right)$ de la vegetación leñosa fue similar a la registrada en bosques maduros Pinus-Quercus del noreste de México. Jiménez et al. (2001) registraron 9 especies, González et al. (2008) 8 especies y Alanís et al. (2008) 7 especies, lo que indica que 10 años después de un incendio existe similitud en riqueza específica de especies leñosas con un ecosistema maduro. La exposición NO presentó menor riqueza específica que la orientación $\mathrm{NE}$, pero mayor diversidad debido a que las especies presentan mayor similitud en las abundancias relativas. De acuerdo con el coeficiente de similitud de Sorensen para datos cuantitativos, las zonas evaluadas (NE vs. NO) mostraron una similitud baja-media (44\%), al presentar únicamente 5 especies en común.

Las conclusiones principales de la investigación son: 1) la existencia de una capacidad de rebrote dada por la alta presencia y dominancia de especies arbóreas favorece la recuperación del estrato arbóreo existente previo a los incendios; 2) la disminución/eliminación de la presencia de la especie de Pinus pseudostrobus en las zonas quemadas. Si bien la especie está adaptada al fuego mediante una corteza gruesa y un banco de semillas longevo, incendios frecuentes de intensidad media y alta implican una notable disminución de la especie; 3) la ausencia de diferencias entre las 2 laderas evaluadas en cuanto a riqueza, diversidad, densidad y área basal, a pesar de las diferencias en orientación entre ellas, indica que la regeneración de la comunidad vegetal estudiada tras el fuego a medio plazo no es afectada por variaciones microclimáticas asociadas a los cambios en orientación, lo que puede sugerir también una gran resiliencia al efecto de la variación climática; 4) no obstante, las 2 exposiciones presentan diferencias importantes en la composición del compartimento leñoso reflejando respuestas a esas variaciones microclimáticas entre laderas a nivel de especie y 5) de acuerdo con el análisis de componentes principales para la densidad y dominancia, las parcelas se separan claramente en fun- ción de su orientación. Con los resultados de la presente investigación, se recomienda la generación de estudios encaminados a evaluar acciones de restauración que incorporen ecotecnologías para la reforestación de la especie clave de P. pseudostrobus.

\section{Agradecimientos}

Al Consejo Nacional de Ciencia y Tecnología (CONACYT), por la beca otorgada al primer autor. A Lillian Belle Willcockson, Directora del Parque Ecológico Chipinque, por todas las facilidades otorgadas para el establecimiento y ejecución de la investigación; a Glafiro Alanís Flores, por el apoyo en la identificación de las especies. La Fundación CEAM está subvencionada por Generalitat Valenciana, Fundación Bancaja y los proyectos GRACCIE (Consolider-Ingenio 2010) y FEEDBACKS (Prometeo Generalitat Valenciana).

\section{Literatura citada}

Alanís, E., J. Jiménez, D. Espinoza, M. A. González, E. Jurado y O. A. Aguirre. 2008. Monitoreo del estrato arbóreo en un área restaurada post-incendio en el Parque Ecológico Chipinque, México. Revista Chapingo serie Ciencias Forestales y del Ambiente 14:113-118.

Alanís, E., J. Jiménez, M. Pando, O. A. Aguirre, E. J. Treviño y P. C. García. 2010. Efecto de la restauración ecológica post-incendio en la diversidad y estructura del componente arbóreo del Parque Ecológico Chipinque, México. Madera y Bosques 16:39-54.

Alanís, E., J. Jiménez, A. Valdecantos, M. Pando, O. Aguirre y E. J. Treviño. 2011. Caracterización de regeneración leñosa post-incendio de un ecosistema templado del Parque Ecológico Chipinque, México. Revista Chapingo. Serie Ciencias Forestales y del Ambiente 17:31-39.

Baeza, M. J. y J. Roy. 2008. Germination of an obligate seeder (Ulex parviflorus) and consequences for wildfire management. Forest Ecology and Management 256:685693.

Calvo, L., S. Santalla, L. Valbuena, E. Marcos, R. Tárrega y E. Luis-Calabuig. 2008. Post-fire natural regeneration of a Pinus pinaster forest in NW Spain. Plant Ecology 197:8190.

Certini, G. 2005. Effects of fire on properties of forest soils: a review. Oecologia 143:1-10.

Clifford, H. y W. Stephenson. 1975. An introduction to numerical classification. Academic, London. 229 p.

Fulé, P. Z. y W. W. Covington. 1999. Fire regime changes in La Michilía Biosphere Reserve, Durango, Mexico. Conservation Biology 13:640-652.

Fulé, P. Z., J. Villanueva-Díaz y M. Ramos-Gómez. 2005. Fire 
regime in a conservation reserve in Chihuahua, Mexico. Canadian Journal of Forest Research 35:320-330.

García, D. A. 2000. Restauración de la vegetación en los bosques de encino y pino en el Parque Ecológico Chipinque, México. Tesis, Facultad de Ciencias Forestales, Universidad Autónoma de Nuevo León, Monterrey. 81 p.

González, M. A., L. Schwendenmann, J. Jiménez y R. Schulz. 2008. Forest structure and woody plant species composition along a fire chronosequence in mixed pine-oak forest in the sierra Madre Oriental, northeast Mexico. Forest Ecology and Management 256:161-167.

González, M. A., L. Schwendenmann, J. Jiménez y W. Himmelsbach. 2007. Reconstrucción del Historial de incendios y estructura forestal en bosques mixtos de pinoencino en la Sierra Madre Oriental. Madera y Bosques 13:51-63.

Himmelsbach, W., E. J. Garza, H. G. Rodríguez, M. A. González, A. E. Estrada y O. A. Aguirre. 2010. Site-conditions reflected by seasonal and diurnal leaf water potentials of four co-occurring tree species in the Sierra Madre Oriental, Mexico. Forstarchiv 81:110-117.

Iniguez, J. M., T. W. Swetnam y S. R. Yool. 2008. Topography affected landscape fire history patterns in southern Arizona, USA. Forest Ecology and Management 256:295-303.

Jiménez, J. y E. Alanís. 2011. Análisis de la frecuencia de los incendios forestales en la sierra Madre Oriental y Occidental del norte de México y sur de Estados Unidos de América. Ciencia UANL 14:255-263.

Jiménez, J., O. A. Aguirre y H. Kramer. 2001. Análisis de la estructura horizontal y vertical en un ecosistema multicohortal de pino-encino en el noreste de México. Investigación Agraria. Sistemas de Recursos Forestales 10:355-366.

Konstantinidis, P., G. Tsiourlis y P. Xofis. 2006. Effect of fire season, aspect and pre-fire plant size on the growth of Arbutus unedo L. (strawberry tree) resprouts. Forest Ecology and Management 225:359-367.

Magurran, A. 2004. Measuring Biological Diversity. Blackwell Science, Oxford. 256 p.

Marroquín, R. A., J. Jiménez, F. Garza, O. A. Aguirre, E. Estrada y R. Bourguet. 2007. Regeneración natural de Pinus pseudostrobus en zonas degradadas por incendio. Ciencia UANL 10:33-37.

Martínez, H. C. y D. A. Rodríguez. 2008. Species diversity after prescribed burns at different intensities and seasons in a high altitude Pinus hartwegii forest. Interciencia 33:337344.

Müeller-Dombois, D. y H. Ellenberg. 1974. Aims and methods of vegetation ecology. John Wiley, New York. 547 p.

Otto, R., B. O. Krüsi, J. D.Delgado, J. M. Fernández-Palacios, E. García-del-Rey y J. R. Arevalo. 2010. Regeneration niche of the Canarian juniper: the role of adults, shrubs and environmental conditions. Annals of Forest Science, DOI: $10.1051 /$ forest/2010034.

Pausas, J. G. 2004. La recurrencia de incendios en el monte mediterráneo. In Avances en el estudio de la gestión del monte mediterráneo, R. V. Vallejo y J. A. Alloza (eds.). Fundación Centro de Estudios Ambientales del Mediterráneo, Valencia. p. 53.

Pausas, J. G., R. A. Bradstock, D. A. Keith y J. E. Keeley. 2004. Plant functional traits in relation to fire in crow-fire ecosystems. Ecology 85:1085-1100.

Richardson, D. M. 2000. Ecology and biogeography of Pinus. Cambridge University Press. 527 p.

Rodríguez, D. A. 2008. Fire Regimes, Fire Ecology, and Fire Management in Mexico. AMBIO: a Journal of the Human Environment 37:548-556.

Rodríguez-Trejo, D. A. y P. Z. Fulé. 2003. Fire ecology of Mexican pines and a fire management proposal. International Journal of Wildland Fire 12:23-37.

Rodríguez-Trejo, D. A. y R. L. Myers. 2010. Using oak characteristics to guide fire regime restoration in Mexican pine-oak and oak forests. Ecological Restoration 28:304323.

Scott, J. H. y E. D. Reinhardt. 2001. Assessing crown fire potential by linking models of surface and crown fire behavior. Research paper RMRS-29. USDA Forest Service, Rocky Mountain Research Station, Fort Collins, Colorado.

Shannon, C. E. 1948. A mathematical theory of communication. Bell System Technical Journal 27:397-423 and 623-656.

van de Water, P. K., S. W. Leavitt y J. L. Betancourt. 2002. Leaf $\mathrm{d}^{13} \mathrm{C}$ variability with elevation, slope aspect, and precipitation in the southwest United States. Oecologia 132:332-343.

Vidal, O. J. y A. Reif. 2011. Effect of a tourist-ignited wildfire on Nothofagus pumilio forests at Torres del Paine biosphere reserve, Chile (Southern Patagonia). Bosque 32:64-67.

Villarreal, M. L. y S. R. Yool. 2008. Analysis of fire-related vegetation patterns in the Huachuca Mountains, Arizona, USA, and Sierra los Ajos, Sonora, Mexico. Fire Ecology 4:14-33.

Weiguo, S., C. Sha y L. Guangqi. 2008. Dynamics of leaf area index and canopy openness of three forest types in a warm temperate zone. Frontiers of Forestry in China 3:416-421.

Woods, S. W., A. Birkas y R. Ahl. 2007. Spatial variability of soil hydrophobicity after wildfires in Montana and Colorado. Geomorphology 86:465-479.

Yocom, L. L., P. Z. Fulé, P. M. Brown, J. N. Cerano, J. Villanueva-Díaz, D. A. Falk y E. Cornejo-Oviedo. 2010. El Niño-Southern Oscillation effect on a fire regime in northeastern Mexico has changed over time. Ecology 91:1660-1671. 